\title{
Myocardial perfusion scintigraphy in the detection of silent ischemia in asymptomatic diabetic patients*
}

\author{
Cintilografia de perfusão miocárdica na detecção da isquemia silenciosa em pacientes diabéticos \\ assintomáticos
}
Gláucia Celeste Rossatto Oki ${ }^{1}$, Elizabeth João Pavin ${ }^{2}$, Otávio Rizzi Coelho ${ }^{3}$, Maria Cândida R. Parisi ${ }^{2}$, Raitany C. Almeida ${ }^{4}$, Elba Cristina Sá de Camargo Etchebehere ${ }^{5}$, Edwaldo Eduardo Camargo ${ }^{6}$, Celso Dario Ramos $^{7}$

\begin{abstract}
Objective: This study was aimed to evaluate myocardial perfusion in asymptomatic patients with type 1 (DM1) and type 2 diabetes mellitus (DM2) without previous diagnoses of coronary artery disease (CAD) or cerebral infarction. Materials and Methods: Fifty-nine consecutive asymptomatic patients (16 DM1, 43 DM2) underwent myocardial perfusion scintigraphy with ${ }^{99 \mathrm{~m}} \mathrm{Tc}$-sestamibi (MPS). They were evaluated for body mass index, metabolic control of DM, type of therapy, systemic arterial hypertension, dyslipidemia, nephropathy, retinopathy, peripheral neuropathy, smoking, and familial history of CAD. Results: MPS was abnormal in 15 patients (25.4\%): 12 (20.3\%) with perfusion abnormalities, and 3 with isolated left ventricular dysfunction. The strongest predictors for abnormal myocardial perfusion were: age 60 years and above $(p=0.017$; odds ratio $[\mathrm{OR}]=6.0)$, peripheral neuropathy $(p=0.028$; OR $=6.1)$, nephropathy $(p=0.031 ; \mathrm{OR}=5.6)$, and stress ECG positive for ischemia $(p=0.049 ; \mathrm{OR}=4.08)$. Conclusion: Silent myocardial ischemia occurs in more than one in five asymptomatic diabetic patients. The strongest predictors of ischemia in this study were: patient age, peripheral neuropathy, nephropathy, retinopathy and a stress ECG positive for ischemia.

Keywords: Diabetes mellitus; Myocardial perfusion imaging; Silent myocardial ischemia; Coronary artery disease.
\end{abstract}

Resumo Objetivo: Este estudo teve por finalidade avaliar a perfusão miocárdica de pacientes com diabetes mellitus tipo 1 (DM1) e tipo 2 (DM2) assintomáticos, sem diagnóstico prévio de doença arterial coronariana (DAC) ou acidente vascular cerebral. Materiais e Métodos: Cinquenta e nove pacientes consecutivos (16 DM1, 43 DM2) foram submetidos a cintilografia de perfusão miocárdica com sestamibi- ${ }^{99 m} \mathrm{Tc}$ (CPM). Foram avaliados quanto ao índice de massa corpórea, controle metabólico do diabetes, dislipidemia, terapia para o diabetes, hipertensão arterial sistêmica, nefropatia, retinopatia, neuropatia periférica, tabagismo e história familiar de DAC. Resultados: CPM foi anormal em 25,4\%: 12 (20,3\%) com alterações de perfusão e 3 com disfunção ventricular esquerda isolada. Os mais fortes preditores de perfusão miocárdica anormal foram: idade igual ou maior a 60 anos ( $p=0,017$, odds ratio $[\mathrm{OR}]=6,0)$, neuropatia periférica $(p=0,028$, OR $=6,1)$, nefropatia $(p=0,031$, OR $=5,6)$ e ECG de esforço positivo para isquemia $(p=$ 0,049, OR = 4,08). Conclusão: A isquemia miocárdica silenciosa ocorre em mais de um em cada cinco diabéticos assintomáticos. Os mais fortes preditores de isquemia foram: idade avançada, neuropatia periférica, nefropatia, retinopatia e ECG de esforço positivo para isquemia.

Unitermos: Diabetes mellitus; Cintilografia de perfusão miocárdica; Isquemia miocárdica silenciosa; Doença arterial coronariana.

Oki GCR, Pavin EJ, Coelho OR, Parisi MCR, Almeida RC, Etchebehere ECSC, Camargo EE, Ramos CD. Myocardial perfusion scintigraphy in the detection of silent ischemia in asymptomatic diabetic patients. Radiol Bras. $2013 \mathrm{Jan} / \mathrm{Fev} ; 46(1): 7-14$.

\footnotetext{
* Study developed at the Division of Nuclear Medicine, Department of Radiology, and Divisions of Endocrinology and Cardiology, Department of Internal Medicine, School of Medical Sciences, Universidade Estadual de Campinas (Unicamp), Campinas, SP, Brazil.

1. Master, Physician Assistant at Serviços de Medicina Nuclear, Clínica Diagnoson and Hospital Aristides Maltez, Salvador, BA, Brazil.

2. PhDs, Physicians Assistant at Service of Endocrinology, Department of Internal Medicine, Universidade Estadual de Campinas (Unicamp), Campinas, SP, Brazil.

3. PhD, MD, Coordinator for the Service of Cardiology, Department of Internal Medicine, Universidade Estadual de Campinas (Unicamp), Campinas, SP, Brazil.

4. Master, Physician at the Service of Cardiology, Department of Internal Medicine, Universidade Estadual de Campinas (Unicamp), Campinas, SP, Brazil.
}

5. PhD, Physician Assistant at the Service of Nuclear Medicine, Department of Radiology, Universidade Estadual de Campinas (Unicamp), Campinas, SP, Brazil.

6. PhD, MD, Coordinator for the Service of Nuclear Medicine, Hospital Sírio-Libanês, Campinas, SP, Brazil.

7. PhD, MD, Coordinator for the Service of Nuclear Medicine, Department of Radiology, Universidade Estadual de Campinas (Unicamp), Campinas, SP, Brazil.

Corresponding author: Dr. Celso Dario Ramos. Avenida Vital Brasil, 251, Cidade Universitária "Zeferino Vaz", Barão Geraldo. Caixa Postal 6142, Campinas, SP, 13083-970, Brazil. E-mail: cdramos@unicamp.br

Received March 11, 2012. Accepted after revision November 19, 2012.

\section{INTRODUCTION}

Coronary artery disease (CAD) is the leading cause of mortality in diabetic patients. In these individuals, CAD is usually more advanced at the time of diagnosis and frequently presents an unfavorable prognosis. In addition, patients with diabetes have a high incidence of occult CAD, observed as an increased occurrence of silent myocardial infarction (SMI) and not typically associated with angina, probably because 
of diabetic autonomic neuropathy ${ }^{(1,2)}$. These factors have raised the interest in investigating CAD before its first clinical expression in diabetic patients and noninvasive tests have been advocated as an essential tool for early detection of the disease $^{(3)}$

In general, individuals with diabetes have at least a twofold to fourfold increased risk for cardiovascular events when compared with age-matched subjects without diabetes. It is estimated that SMI prevalence in this group ranges from $10 \%$ to $20 \%$ versus from $1 \%$ to $4 \%$ estimated for non diabetic populations ${ }^{(\mathbf{4})}$. After myocardial infarction, diabetic patients have a twofold to threefold greater morbidity and mortality. The high risk of CAD in these patients took the American Heart Association to not only determine diabetes as a major independent risk factor for the development of CAD as to establish that diabetes is a cardiovascular disease ${ }^{(5)}$.

Asymptomatic diabetic patients with increased risk for CAD can benefit of myocardial perfusion scintigraphy with ${ }^{99 \mathrm{~m}} \mathrm{Tc}$ sestamibi (MPS) because this scintigraphic test cost-effectively measures and stratifies risk. Therefore this test is a good alternative to cineangiocoronariography or treadmill test ${ }^{(\mathbf{6})}$. Even so, many authors have been using cineangiocoronariography as gold standard for $\mathrm{CAD}^{(\mathbf{3}, 7)}$. However, those studies do not take into account the microvascular pathophysiology of diabetes mellitus for which cineangiocoronariography and stress electrocardiogram (ECG) have a lower sensitivity ${ }^{(\mathbf{5})}$. Indeed, there is consistent evidence that diabetes causes alterations in the regulation of coronary vasodilator function in both epicardial and resistance coronary vessels, which are present before the appearance of obstructive $\mathrm{CAD}^{(\mathbf{8})}$. Thus, functional imaging can more accurately identify endothelial cell dysfunction within coronary microvasculature among diabetic patients.

Considering that MPS is relatively expensive to be used as a screening test, it is necessary to select subgroups of diabetic patients who present an increased risk for CAD, and that will effectively benefit of systematic screening for myocardial perfusion abnormalities. This study was aimed to evaluate myocardial perfusion in dia- betic patients type 1 (DM1) and type 2 (DM2) without previous diagnoses or symptoms of CAD and cerebral infarction, as well as to evaluate clinical and laboratory predictors of abnormal test results.

\section{MATERIALS AND METHODS}

The study was approved by the ethics committee of the institution and all patients signed a written consent form. The authors do not have any conflict of interest in this project.

During a 12-month period, consecutive patients who met the inclusion criteria were invited by endocrinologists during their clinical appointments to participate in the protocol. Fifty-nine patients with the diagnosis of DM (16 type 1 and 43 type 2 ) were prospectively studied (44 women and 15 men; ages 26 to 74 years; mean $49.4 \pm 12.2$ years). Disease duration was $14.3 \pm 7.2$ years. Inclusion criteria were: diagnosis of type 1 diabetes or type 2 diabetes (according to American Diabetes Association criteria) ${ }^{(\mathbf{9})}$; age between 18 and 75 years. Exclusion criteria were: presence of clinical symptoms or ECG signs of CAD, history of cerebral infarction or myocardial infarction, coronary revascularization or heart failure, and pregnancy.

Patients were clinically evaluated by the endocrinologist and the nuclear medicine physician about sex, age, type of diabetes, disease duration, treatment (insulin, insulin plus oral agents or oral agents), metabolic control of diabetes, systemic arterial hypertension $(\geq 140 \mathrm{mmHg}$ systolic blood pressure and/or $\geq 90 \mathrm{mmHg}$ diastolic blood pressure) or treatment with an antihypertensive drug, smoking, body mass index (BMI) $\left(\mathrm{kg} / \mathrm{m}^{2}\right)$, regular physical activity, familial history of CAD, dyslipidemia or hypolipemic treatment, and presence of diabetic nephropathy, retinopathy, and peripheral neuropathy. The laboratory evaluation included: hemoglobin A1c level (good control $\mathrm{HbA}_{1 \mathrm{c}}<7 \%$ ), total serum cholesterol (normal value: $<200 \mathrm{mg} / \mathrm{dl}$ ), LDL cholesterol (normal value: $<100 \mathrm{mg}$ / dl), HDL cholesterol (normal value: $>40$ $\mathrm{mg} / \mathrm{dl}$ for males and $>50 \mathrm{mg} / \mathrm{dl}$ for females), triglycerides (normal value: $<150$ $\mathrm{mg} / \mathrm{dl}$ ), albumin to creatinine ratio, as the mean of 3 nonconsecutive morning spot urine samples (microalbuminuria: $\geq 30$ to $299 \mathrm{mg} / \mathrm{g}$ creatinine, and macroalbuminuria: $\geq 300 \mathrm{mg} / \mathrm{g}$ creatinine $)^{(\mathbf{1 0})}$. Fundoscopy reports by an eye-care professional were evaluated for the presence and stage of diabetic retinopathy. Patients were tested for peripheral neuropathy (sensation to touch by monofilament and vibration sensation by tuning fork and Achilles tendon reflex by reflex hammer). Clinical findings are summarized in Table 1.

\section{Stress SPECT myocardial perfusion imaging}

All patients underwent MPS. Patients were oriented to suspend xanthines and nitrates one day before, beta-blockers three days before, and calcium channel blockers five days before the scintigraphy. Rest and stress imaging were performed on the same day. First it was acquired resting tomographic images (SPECT) after the injection of $10 \mathrm{mCi}(370 \mathrm{MBq})$ of sestamibi- ${ }^{99 \mathrm{~m}} \mathrm{Tc}$ with the following parameters: high-resolution collimator, matrix $64 \times 64$, angle range $186^{\circ}$, angle step $6^{\circ}$. Patients were then submitted to stress by the cardiologist (exercise or pharmacological stress with dipyridamole) and received an injection of $30 \mathrm{mCi}(1110 \mathrm{MBq})$ at stress peak. Later acquisition of stress imaging followed the same gamma-camera parameters but it was also synchronized to the ECG (GATED SPECT). The left ventricle ejection fraction (LVEF) derived from these ECG-gated images. In the course of scintigraphy, electrocardiograms were recorded, at rest and after stress, and analyzed by the cardiologist. All patients who presented perfusion defects in the inferior wall at the stress imaging repeated the acquisition in prone position to evaluate diaphragmatic artifacts. Scintigraphies were visually analyzed by two nuclear medicine physicians and, if abnormal, quantified with the 17segment method ${ }^{(11)}$.

When necessary, semiquantitative 17segment visual interpretation was performed using a 5-point scoring system $(0$, normal; 1 , mild; 2 , moderate; 3 , severe reduction of radioisotope uptake; and 4 , absence of detectable tracer uptake). The summed stress score and summed rest score were obtained by adding the scores of the 17 segments of the respective im- 
Table 1 Clinical characteristics of the 59 patients.

\begin{tabular}{lcc}
\hline \multicolumn{1}{c}{ Characteristics } & Type 2 diabetes & Type 1 diabetes \\
\hline$n$ & 43 & 16 \\
Sex (male / female) & 10 male / 33 female & 5 male / 11 female \\
Age (years) & $54.7 \pm 8.9(34$ to 74$)$ & $35.1 \pm 7.6(26$ to 50) \\
Body mass index $\left(\mathrm{kg} / \mathrm{m}^{2}\right)$ & $30.9 \pm 6.3$ & $27.0 \pm 5.2$ \\
Duration of diabetes (years) & $13.6 \pm 7.7$ & $16.3 \pm 5.9$ \\
Treatment & & \\
$\quad-$ Oral agent (\%) & $34.8(15 / 43)$ & $0(0 / 16)$ \\
$\quad-$ Insulin plus oral agent (\%) & $51.2(22 / 43)$ & $12.5(2 / 16)$ \\
$\quad-$ Insulin (\%) & $14.0(6 / 43)$ & $87.5(14 / 16)$ \\
HbA ${ }_{1 \mathrm{c}}$ (\%) & $8.8 \pm 1.8$ & $9.1 \pm 1.9$ \\
Retinopathy (\%) & $48.8(21 / 43)$ & $50(8 / 16)$ \\
Nephropathy (\%) & & $12.5(2 / 16)$ \\
$\quad-$ Microalbuminuria & $23.2(10 / 43)$ & $25(4 / 16)$ \\
$\quad-$ Macroalbuminuria & $27.9(12 / 43)$ & $43.7(7 / 16)$ \\
Neuropathy (\%) & $55.8(24 / 43)$ & $18.7(3 / 16)$ \\
Smokers (\%) & $20.9(9 / 43)$ & $62.5(10 / 16)$ \\
Dyslipidemia (\%) & $79.0(34 / 43)$ & $62.5(10 / 16)$ \\
Hypertension (\%) & $90.6(39 / 43)$ & $25(4 / 16)$ \\
Family history of CAD & $41.8(18 / 43)$ & \\
\hline
\end{tabular}

Data are means \pm standard deviation or $\%$ of affected patients. CAD, coronary arterial disease.

ages. The sum of the differences between each of the 17 segments from these images was defined as the summed difference score, representing the amount of ischemia. Each of these variables incorporates the extent and severity of perfusion defects, which independently add prognostic information. These indices were converted to percent of the total myocardium involved with stress, ischemic, or fixed defects by dividing the summed scores by 68 , the maximum potential score in the 17-segment model $(4 \times 17)$, and multiplying by $100^{(\mathbf{1 1})}$.

\section{Statistical analyses}

Statistical analyses were performed with SAS software (SAS Institute Inc.; Cary, NC, USA). First, data from both groups (DM1 and DM2) were statistically analyzed together, using descriptive statistics (mean, standard deviation, minimum, median, and maximum) for continuous variables, and tables of frequencies for categorical variables. Chi-square test and Fisher's exact test were used to compare the categorical variables between the two groups (normal MPS and abnormal MPS) and Mann-Whitney test was used to compare the numerical variables due to the lack of normal distribution of the variables. To determine independent risk factors related with ischemia and/or fixed defect a logistic regression analysis was performed, using univariate and multiple models, with stepwise criterion for variable selection. A $\mathrm{p}$ value $<0.05$ was considered significant. After this first evaluation, data from the DM2 group was analyzed separately, also using the descriptive statistics and the same tests.

The age cutoff according to the presence of abnormalities in MPS, in order to maximize sensitivity and specificity, was determined by receiver operating characteristic (ROC) curve analysis.

\section{RESULTS}

MPS was abnormal in $15 / 59$ patients (25.4\%): 12 with perfusion defects (2/16 DM1 and 10/43 DM2) and 3 with left ventricular dysfunction, defined by ejection fraction under 45\% (3/43 DM2). For statistical analyses, it was considered as abnormal only the group with perfusion defects $(n=12)$, since isolated left ventricular dysfunction could represent diabetic or hypertensive cardiomyopathy instead of true silent ischemia. Therefore in our study the term "silent ischemia" would best describe patients with myocardial perfusion abnormalities.
Among patients with abnormal perfusion tests $(n=12)$, we had 2 with fixed defects and 10 with ischemia. Considering the 17-segment quantification ${ }^{(\mathbf{1 1})}$ and establishing a cut off of $10 \%$, we had 7 low risk cases (quantification $<10 \%$ ), and 5 high risk cases (quantification $\geq 10 \%$ ) including 1 patient with TID (transient ischemic dilation) which was also considered high risk even though it was not possible to quantify it by the same method. Still considering these subgroup, we had 3 patients with left ventricular dysfunction ( $\mathrm{LVEF}<45 \%$ ) associated to the perfusion defect (Table 2).

In this subgroup we also observed 5 patients with ischemic stress tests ( 1 exercise and 4 dipyridamole), and 7 patients with normal stress tests ( 3 exercise and 4 dipyridamole). On the other hand in the normal MPS group, 41 patients had normal stress tests (28 exercise and 13 dipyridamole), and 6 patients had ischemic stress tests (4 exercise and 2 dipyridamole).

The ROC curve analysis for age, using the presence of silent ischemia as the gold standard, revealed the age of 60 years as the cutoff that maximizes sensitivity and specificity. Similarly, comparative analysis of age (continuous variable) between groups with and without silent ischemia in the total sample showed a median of 58.0 years for the group of patients with perfusion abnormalities ( $p=0.026$, Mann Whitney's test).

The prevalence of positive screening tests to silent ischemia varied according to type of diabetes. In the type 1 diabetes group, 2 of 16 patients $(12.5 \%)$ had abnormal perfusion tests (both women, 35 and 46 years old, and duration of diabetes of 17 and 18 years respectively). The stress ECG was normal in these 2 patients. Retinopathy, nephropathy and peripheral neuropathy were present in the first patient and were absent in the other patient. In the type 2 diabetes group, 10 of 43 patients (23.25\%), including 4 men and 6 women, had positive tests (2 fixed defect and 8 ischemic). The ages of patients had ranged between 46 to 74 years and the maximum time of duration of disease was 28 years. The stress ECG was abnormal in half of these 10 patients. Unlikely the DM1 group, retinopathy and nephropathy were present in $80 \%(8 / 10)$ and neuropathy was present in $90 \%(9 / 10)$ 
Table 2 Characteristics of 12 patients with perfusion defects.

\begin{tabular}{|c|c|c|c|c|c|c|c|c|c|c|}
\hline Patient & Sex & $\begin{array}{c}\text { Age } \\
\text { (years) }\end{array}$ & $\begin{array}{l}\text { Type } \\
\text { of DM }\end{array}$ & Nephropathy & Retinopathy & Neuropathy & $\begin{array}{l}\text { Stress } \\
\text { ECG }\end{array}$ & $\begin{array}{l}\text { LVEF } \\
(\%)\end{array}$ & MPS & $\begin{array}{c}\text { Quantification* } \\
\text { (\%) }\end{array}$ \\
\hline 1 & Female & 46 & 1 & Macroalbuminuria & Yes & Yes & Normal & 52 & Ischemia & 7 \\
\hline 2 & Female & 35 & 1 & No & No & No & Normal & 56 & Ischemia & 6 \\
\hline 3 & Female & 58 & 2 & No & No & Yes & Normal & 69 & Ischemia & 6 \\
\hline 4 & Female & 46 & 2 & Macroalbuminuria & Yes & Yes & Abnormal & 43 & Ischemia & 6 \\
\hline 5 & Male & 74 & 2 & Microalbuminuria & Yes & Yes & Abnormal & 52 & Ischemia & 7 \\
\hline 6 & Female & 61 & 2 & Microalbuminuria & Yes & Yes & Abnormal & 56 & Ischemia & 37 \\
\hline 7 & Male & 46 & 2 & Macroalbuminuria & Yes & Yes & Abnormal & 39 & Fixed defect & 12 \\
\hline 8 & Female & 55 & 2 & Microalbuminuria & Yes & Yes & Normal & 55 & Ischemia & 12 \\
\hline 9 & Male & 66 & 2 & Macroalbuminuria & Yes & Yes & Normal & 52 & Ischemia & 6 \\
\hline 10 & Female & 68 & 2 & Macroalbuminuria & Yes & Yes & Normal & 47 & Fixed defect & 12 \\
\hline 11 & Female & 58 & 2 & No & Yes & Yes & Normal & $>70$ & TID & - \\
\hline 12 & Male & 72 & 2 & Macroalbuminuria & No & No & Abnormal & 33 & Ischemia & 9 \\
\hline
\end{tabular}

DM, diabetes mellitus; LVEF, left ventricular ejection fraction; MPS, myocardial perfusion scintigraphy; TID, transient ischemic dilation.

* Quantification with the 17-segment method ${ }^{(12)}$.

of DM2 patients with perfusion abnormalities. However, these different rates of prevalence between the diabetic groups (type 1 and type 2) could not be statistically analyzed due to the reduced number of DM1 patients included in the study (Table 2).

Considering the 12 patients with abnormal MPS (2 DM1 and 10 DM2), statistical analyses of categorical variables using Chi-square test and Fisher's exact test revealed to be statistically significant predictors of the presence of silent ischemia in diabetics patients: age 60 years and above $(p=0.022)$, retinopathy $(p=0.045)$, nephropathy $(p=0.032)$, and peripheral neuropathy ( $p=0.017$ ) (Table 3$)$.

Conversely, no difference was observed between the two groups, with and without ischemia, regarding gender, BMI, duration of diabetes, type of treatment, $\mathrm{HbA}_{1 \mathrm{c}}$ level, dyslipidemia, arterial hypertension, smoking, abnormal stress ECG, physical activity or familial history of DAC.

Univariate logistic regression analysis showed to be statistically significant predictors of silent ischemia: age 60 years and above $(p=0.017$; odds ratio $[\mathrm{OR}]=6.0)$, abnormal stress ECG ( $p=0.049$; OR = $4.08)$, nephropathy $(p=0.042)$; OR = 4.42 ), and peripheral neuropathy ( $p=$ 0.028 ; OR = 6.19). Additionally, a significant correlation was observed between silent ischemia and macroalbuminuric nephropathy ( $p=0.031$; OR $=5.60)$ (Table 4$)$.

Multiple logistic regression analysis showed that the variables which better in-
Table 3 Clinical characteristics of patients with or without silent coronary disease.

\begin{tabular}{|c|c|c|c|}
\hline Characteristics & $\begin{array}{l}\text { Abnormal } \\
\text { perfusion }\end{array}$ & $p$ value & $\begin{array}{l}\text { Normal } \\
\text { perfusion }\end{array}$ \\
\hline$n$ & 12 & & 47 \\
\hline Sex (male/female) & $4 / 8$ & NS & $11 / 36$ \\
\hline Age $(<60 / \geq 60$ years $)$ & $7 / 5$ & $0.022 *$ & $42 / 5$ \\
\hline Type of DM $(1 / 2)$ & $2 / 10$ & NS & $14 / 33$ \\
\hline Body mass index $\left(<25 / \geq 25 \mathrm{~kg} / \mathrm{m}^{2}\right)$ & $1 / 11$ & NS & $10 / 37$ \\
\hline Duration of disease $(<15 / \geq 15$ years $)$ & $5 / 7$ & NS & $26 / 21$ \\
\hline Use of insulin (Yes/No) & $9 / 3$ & NS & $35 / 12$ \\
\hline $\mathrm{HbA}_{1 \mathrm{c}}(<7 / \geq 7 \%)$ & $3 / 9$ & NS & $7 / 40$ \\
\hline Retinopathy (Yes/No) & $9 / 3$ & $0.045^{\dagger}$ & $20 / 27$ \\
\hline Nephropathy (Yes/No) & $9 / 3$ & $0.032^{\dagger}$ & $19 / 28$ \\
\hline - Microalbuminuria & $3 / 9$ & NS & $9 / 19$ \\
\hline - Macroalbuminuria & $6 / 9$ & NS & $10 / 19$ \\
\hline Neuropathy (Yes/No) & $10 / 2$ & $0.017^{\dagger}$ & $21 / 26$ \\
\hline Smokers (Yes/No) & $4 / 8$ & NS & $8 / 39$ \\
\hline Hypertension (Yes/No) & $12 / 0$ & NS & $37 / 10$ \\
\hline Dyslipidemia (Yes/No) & $9 / 3$ & NS & $35 / 12$ \\
\hline Familial history of CAD (Yes/No) & $4 / 8$ & NS & $18 / 29$ \\
\hline Regular physical activity (Yes/No) & $3 / 9$ & NS & $8 / 39$ \\
\hline Stress ECG positive for ischemia (Yes/No) & $5 / 7$ & NS & $7 / 40$ \\
\hline
\end{tabular}

NS, non significant; DM, diabetes mellitus; CAD, coronary arterial disease.

* Fisher's exact test $(p$ value $<0.05) ;{ }^{\dagger}$ Chi-square test $(p$ value $<0.05)$.

Table 4 Univariate logistic regression analysis for ischemia ( $p$ value $<0.05$ ).

\begin{tabular}{lccccc}
\hline & $\begin{array}{c}\text { MPS } \\
\text { abnormal } \\
n=12\end{array}$ & $\begin{array}{c}\text { MPS } \\
\text { normal } \\
n=47\end{array}$ & $p$ value & OR & Cl 95\% OR \\
\hline $\begin{array}{l}\text { Age }(<60 / \geq 60 \text { years) } \\
\text { Stress ECG positive for }\end{array}$ & $7 / 5$ & $42 / 5$ & 0.017 & 6.0 & $1.37-26.24$ \\
ischemia (Yes/No) & $5 / 7$ & $7 / 40$ & 0.049 & 4.08 & $1.01-16.56$ \\
Nephropathy (Yes/No) & $9 / 3$ & $19 / 28$ & 0.042 & 4.42 & $1.06-18.48$ \\
- Microalbuminuria & $3 / 9$ & $9 / 19$ & NS & 3.11 & $0.53-18.22$ \\
- Macroalbuminuria & $6 / 9$ & $10 / 19$ & 0.031 & 5.60 & $1.17-26.72$ \\
Neuropathy (Yes/No) & $10 / 2$ & $21 / 26$ & 0.028 & 6.19 & $1.22-31.39$ \\
\hline
\end{tabular}

MPS, myocardial perfusion scintigraphy; OR, odds ratio; $\mathrm{Cl}$, confidence interval; NS, non significant. 

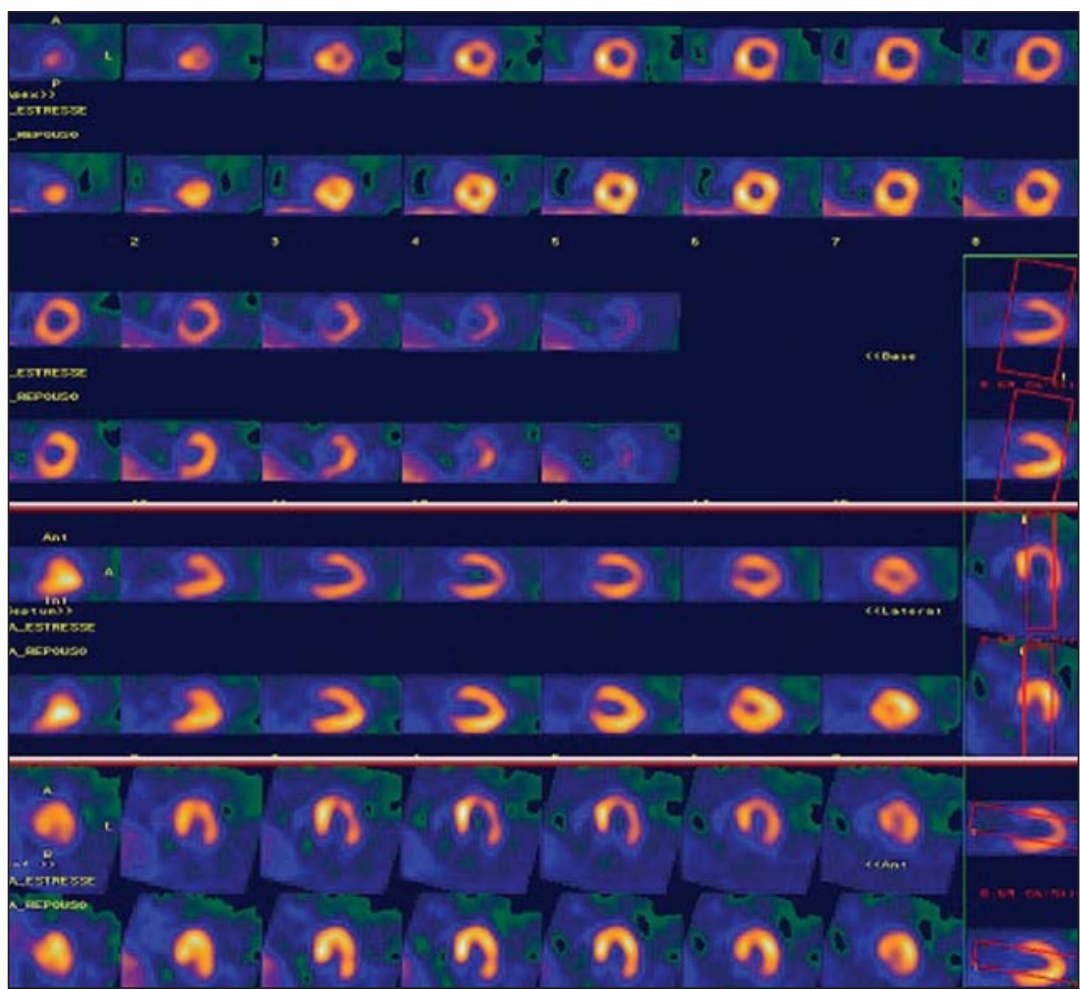

Figure 1. Clinical case 1. A 61-year-old woman with diabetes type 2 for 28 years and clinical manifestations of diabetic nephropathy, retinopathy, and peripheral neuropathy. Patient was asymptomatic. Pharmacological stress with dipyridamole was positive for ischemia. MPS showed ischemia; 17-segment quantification $=37 \%$.
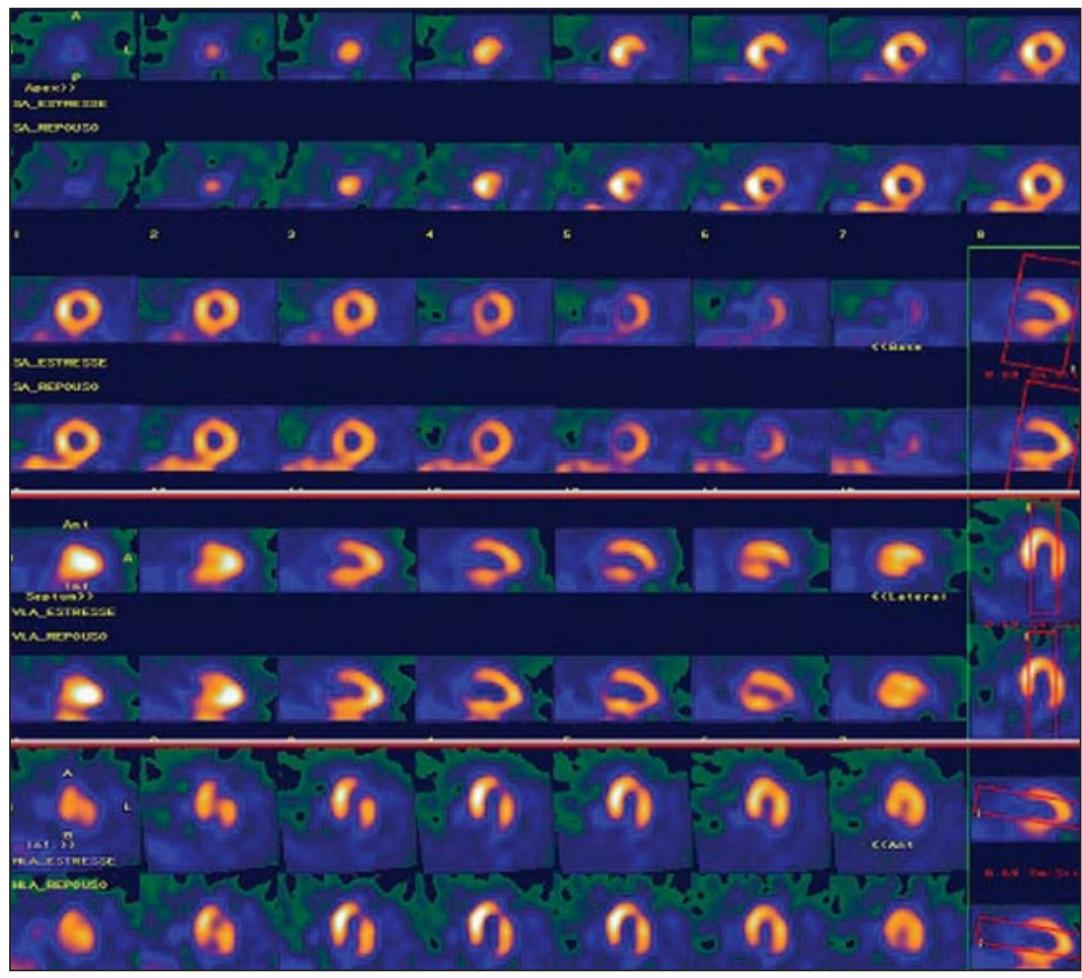

Figure 2. Clinical case 2. A 68-year-old woman with diabetes type 2 for 16 years and clinical manifestations of diabetic nephropathy, retinopathy, and peripheral neuropathy. Patient was asymptomatic. Pharmacological stress with dipyridamole was negative for ischemia. MPS showed fixed defect with ischemic component; 17 -segment quantification $=12 \%$. dicates concomitant silent ischemia in diabetic patients are: age 60 years and above $(p=0.039 ; \mathrm{OR}=5.21)$, and peripheral neuropathy $(p=0.045 ; \mathrm{OR}=5.54)($ Table 5$)$.

As the small number of patients with DM1 included in the study did not allow an individual statistical analysis of this subgroup, we conducted a separate analysis only the subset of patients with DM2.

The ROC curve analysis for age in DM2 patients, using the presence of silent ischemia as the gold standard, revealed the same cutoff of 60 years. Similarly, comparative analysis of age (continuous variable) between groups with and without silent ischemia showed a median of 59.5 years for the group of DM2 patients with perfusion abnormalities ( $p=0.041$; Mann Whitney's test).

Still considering only the $10 \mathrm{DM} 2 \mathrm{pa}-$ tients with abnormal scintigraphy, the statistical analysis of categorical variables using Fisher's exact test revealed to be statistically significant predictors of the presence of silent ischemia in DM2: age 60 years and above $(p=0.036)$, diabetic retinopathy $(p=0.034)$, and peripheral neuropathy $(p=0.026)$. Unlike the statistical analysis of the total sample, which assessed the DM1 and DM2 together, the presence of diabetic nephropathy was not statistically significant when DM2 patients were analyzed separately.

The univariate logistic regression analysis showed as statistically significant predictors of silent ischemia in DM2 patients: age 60 years and above $(p=0.031 ; \mathrm{OR}=$ $5.6)$, diabetic retinopathy ( $p=0.036$; OR $=6.15)$, peripheral neuropathy $(p=0.032$; $\mathrm{OR}=10.8)$, and macroalbuminuric nephropathy ( $p=0.043$; OR = 6.79) (Table 6).

Finally, multiple logistic regression analysis showed that the variable which best indicates possible silent ischemia in DM2 subjects is the presence of peripheral neuropathy $(p=0.032 ; \mathrm{OR}=10.8)$.

\section{DISCUSSION}

Diabetes is associated with a twofold to fourfold risk increase of developing CAD compared with age-matched subjects without diabetes $^{(\mathbf{1 2 - 1 4 )}}$. Many studies have been designed to evaluate the presence of silent myocardial ischemia in asymptomatic dia- 
Table 5 Multiple logistic regression analysis for ischemia ( $p$ value $<0.05$ ).

\begin{tabular}{lccccc}
\hline & $\begin{array}{c}\text { MPS } \\
\text { abnormal } \\
n=12\end{array}$ & $\begin{array}{c}\text { MPS } \\
\text { normal } \\
n=47\end{array}$ & $p$ value & OR & Cl 95\% OR \\
\hline Age $(<60 / \geq 60$ years) & $7 / 5$ & $42 / 5$ & 0.039 & 5.21 & $1.09-24.96$ \\
Neuropathy (Yes/No) & $10 / 2$ & $21 / 26$ & 0.045 & 5.54 & $1.04-29.58$ \\
\hline
\end{tabular}

MPS, myocardial perfusion scintigraphy; OR, odds ratio; $\mathrm{Cl}$, confidence interval.

Table 6 Univariate logistic regression analysis for ischemia in DM2 patients ( $p$ value $<0.05$ ).

\begin{tabular}{cccccc}
\hline \multicolumn{1}{c}{$\begin{array}{c}\text { MPS } \\
\text { abnormal } \\
n=10\end{array}$} & $\begin{array}{c}\text { MPS } \\
\text { normal } \\
n=33\end{array}$ & $p$ value & OR & Cl 95\% OR \\
\hline Age $(<60 / \geq 60$ years) & $5 / 5$ & $28 / 5$ & 0.031 & 5.6 & $1.17-26.72$ \\
Retinopathy (Yes/No) & $8 / 2$ & $13 / 20$ & 0.036 & 6.15 & $1.13-33.67$ \\
Nephropathy (Yes/No) & $8 / 2$ & $14 / 19$ & NS & 5.43 & $0.99-29.61$ \\
- Microalbuminuria & $3 / 8$ & $7 / 14$ & NS & 4.07 & $0.56-29.73$ \\
- Macroalbuminuria & $5 / 8$ & $7 / 14$ & 0.043 & 6.79 & $1.06-43.36$ \\
Neuropathy (Yes/No) & $9 / 1$ & $15 / 18$ & 0.032 & 10.8 & $1.23-95.21$ \\
\hline
\end{tabular}

MPS, myocardial perfusion scintigraphy; OR, odds ratio; $\mathrm{CI}$, confidence interval; NS, non significant.

betic patients and their prevalence rates range from $9 \%$ to $57 \%{ }^{(2,7,15-24)}$. The reason for this broad range is probably due to differences in the populations studied, including age of patients, duration of disease, inclusion and exclusion criteria and definition and diagnosis of SMI. Two relatively recent large retrospective studies from $\mathrm{Ce}$ dars Sinai $(n=826)^{(\mathbf{2 5})}$ and the Mayo Clinic $(n=1427)^{(\mathbf{2 6})}$ reported abnormal stress SPECT images in $39 \%$ and $58 \%$ of asymptomatic diabetic patients, respectively. However, retrospective studies have several limitations, including selection bias of higher-risk patients.

The most important prospective study using stress SPECT imaging, the Detection of Ischemia in Asymptomatic Diabetics (DIAD) study ${ }^{(2)}$, evaluated 1123 patients with type 2 diabetes, aged 50-75 years, with no known or suspected CAD. Those patients were randomly assigned to either stress testing and 5-year clinical follow-up or to follow-up only (no imaging). A total of 113 patients (22\%) had evidence of silent myocardial ischemia, including 83 with regional myocardial perfusion abnormalities and 30 with normal perfusion but other abnormalities (i.e., adenosine-induced ST-segment depression, ventricular dilation, or rest ventricular dysfunction). Moderate or large perfusion defects were present in 33 patients. The strongest predictors for abnormal tests were abnormal Val- salva, male sex, and diabetes duration. Other traditional cardiac risk factors or inflammatory and prothrombotic markers were not predictive. The findings of the first phase of DIAD study suggest that greater than one in five asymptomatic patients with type 2 diabetes, aged 50-75 years, have silent ischemia. Considering that the DIAD patient population was truly representative of asymptomatic patients with type 2 diabetes without any clinical reason to suspect CAD, the prevalence of SMI indicates that totally asymptomatic diabetic patients have at least an intermediate probability of CAD, justifying screening by noninvasive testing such as stress myocardial perfusion imaging ${ }^{(2)}$.

In our study, the prevalence of SMI was $20.3 \%$, which is similar to DIAD results and to several other studies of the literature ${ }^{(2,7,21)}$. Although the prevalence of SMI was different when we compared the DM1 and DM2 groups (12.5\% versus $23.25 \%$, respectively), this difference was not statistically significant, probably due to the small number of type 1 diabetics studied.

The association between autonomic neuropathy and asymptomatic ischemia has been demonstrated by several prior studies ${ }^{\text {(27-29) }}$. Similarly, we did not find significantly association among duration of disease, type of diabetes and treatment, BMI, smoking, physical activity, familial history of DAC, dyslipidemia, arterial hyperten- sion, abnormal stress ECG, $\mathrm{HbA}_{1 \mathrm{c}}$ level and presence of silent ischemia. The univariate and multiple logistic regression analyses revealed that age 60 years and above, but no male sex, was a strong predictive risk factor for myocardial ischemia. Both analyses also showed that peripheral neuropathy in our diabetic patients was significantly correlated to silent ischemia. Although we did not have conducted any tests for detection of autonomic neuropathy, we can consider peripheral neuropathy as a predictor of ischemia, since it is present in most patients with diabetic dysautonomia.

However we had a predominance of female patients, although there are no published data that suggest a higher prevalence of diabetes in this sex. We believe that the larger number of women in our study is related to the greater availability of them to participate in research protocols. A similar number of men and women were invited to participate in the protocol, however, more women accepted the invitation and attended the laboratory on the day of the test. Many male patients who initially agreed to participate, did not attend the appointment.

The progress made in detection and treatment of CAD allows reconsidering the screening of silent ischemia, in the hope that early CAD diagnosis leads to a more effective therapy and the decrease of cardiovascular complications and mortality. The challenge to the physician is to select the patient subgroups more likely to benefit from screening ${ }^{(\mathbf{3 0})}$. Current guidelines recommend screening it in asymptomatic diabetic patients selected for high cardiovascular risk (i.e., with two or more other cardiovascular risk factors, or peripheral or carotid arterial disease, or proteinuria). ECG stress test can be recommended in first intention if maximal heart rate can be achieved. For patient with inconclusive ECG stress test, myocardial scintigraphy seems more accurate than stress echocardiography. Coronary angiogram should be performed in case of positive stress test ${ }^{(31)}$.

The second phase of the DIAD study ( $n$ $=1123$ ) already showed that screening with MPS all asymptomatic diabetic patients is not cost-effective ${ }^{(32)}$. The cumulative cardiac event rate was $2.9 \%$ over a follow-up of $4.8 \pm 0.9$ years for an average of $0.6 \%$ per year. The positive predictive value of 
having moderate or large myocardial perfusion defects was only $12 \%$. Thus routine screening did not appear to affect overall outcome $^{(32)}$. However, during the course of study there was a significant and equivalent increase in primary medical prevention in both groups and that can be an important bias. Nonprotocol stress tests were done during follow-up when clinically indicated in both groups and probably led to more stringent measures to control the DM, thus patients might have presented a better outcome than initially expected. Therefore, the goal is to identify the diabetic subgroups with the highest risk for future cardiac events to offer them more aggressive intensive medical therapy or coronary revascularization and optimum medical therapy ${ }^{(33)}$.

In our series of DM1 and DM2 patients, according to univariate logistic regression analysis, these subgroups would be initially determined by the patient's age ( 60 years and above), presence of peripheral neuropathy, nephropathy, retinopathy, or stress ECG positive for ischemia. When the same patients were evaluated by multiple logistic regression analysis, the best predictors to indicate an abnormal myocardial perfusion scintigraphy would be age, or presence of peripheral diabetic neuropathy.

When considering the univariate logistic regression analysis of the subgroup of DM2 patients, individuals with an increased likelihood of myocardial perfusion defects are those which have age 60 years old and above, presence of retinopathy, peripheral neuropathy, or macroalbuminuric nephropathy. Attempting an even more accurate statistical selection, the multiple logistic regression analysis showed that the main predictor of silent ischemia was the presence of peripheral neuropathy, with a 10.8 times greater risk of silent ischemia in an asymptomatic diabetic patient type 2 .

Interestingly, if we consider that patients with peripheral neuropathy may also present autonomic neuropathy, as both are part of the same complication, our results are in agreement with the findings of the first phase of the DIAD study ${ }^{(2)}$, in which the strongest predictor of silent ischemia was reduced heart rate response to Valsalva maneuver.

Currently there is an even greater concern in reducing as much as possible the ex- posure of patients to ionizing radiation. For such, more modern equipment allow the use of a dose of $5 \mathrm{mCi}(185 \mathrm{MBq})$ of sestamibi- ${ }^{99 \mathrm{~m}} \mathrm{Tc}$ at rest imaging and of $15 \mathrm{mCi}$ (555 MBq) in stress imaging for one-day protocol. Another alternative is to use the two-day protocol with fixed doses of $7 \mathrm{mCi}$ (259 MBq) in each step. Some authors also suggest the possibility of performing first the stress imaging after the injection of 7 $\mathrm{mCi}(259 \mathrm{MBq})$, and the rest imaging only if the stress phase is abnormal ${ }^{(34,35)}$. However, this proposal remains controversial. Another important issue related to MPS is the definition of which patients might benefit from revascularization, considering myocardial viability and contractile reserve $^{(36,37)}$.

Future prospective studies including only those subgroups of diabetic patients at high risk for CAD are needed to assess the real benefit of offering these patients a more aggressive therapy.

\section{CONCLUSION}

In our series, about one in five asymptomatic diabetic patients had silent ischemia. Approximately $40 \%$ of DM1 and DM2 asymptomatic patients with silent ischemia had a high risk of cardiac events, with perfusion defects of $10 \%$ and higher.

The predictors of a myocardial scintigraphy showed perfusion abnormalities were: age 60 years and above; stress ECG positive for ischemia; presence of any vascular complication of diabetes: retinopathy, nephropathy, or peripheral neuropathy. Peripheral neuropathy was the main predictor for silent ischemia in asymptomatic DM2 patients.

It is possible to clinically imply that screening of a subgroup of diabetic patients at high risk could lead to early detection and treatment of CAD, which could be useful in reducing morbidity and mortality of these patients.

\section{Acknowledgements}

This study was developed at the Division of Nuclear Medicine, Department of Radiology, and Divisions of Endocrinology and Cardiology, Department of Internal Medicine, School of Medical Sciences, University of Campinas (Unicamp), Cam- pinas, SP, Brazil as part of a Master's degree thesis and the student scholarship was granted by Coordenação de Aperfeiçoamento de Pessoal de Nível Superior (Capes). The project was also granted by Fundo de Apoio ao Ensino, à Pesquisa e Extensão (Faepex) grant number 193/2009.

\section{REFERENCES}

1. Nesto RW. Screening for asymptomatic coronary artery disease in diabetes. Diabetes Care. 1999; 22:1393-5

2. Wackers FJ, Young LH, Inzucchi SE, et al. Detection of silent myocardial ischemia in asymptomatic diabetic subjects: the DIAD study. Diabetes Care. 2004;27:1954-61.

3. Kang X, Berman DS, Lewin H, et al. Comparative ability of myocardial perfusion single-photon emission computed tomography to detect coronary artery disease in patients with and without diabetes mellitus. Am Heart J. 1999;137:949 57.

4. Mieres JH, Rosman DR, Shaw LJ. The role of myocardial perfusion imaging in special populations: women, diabetics, and heart failure. Semin Nucl Med. 2005;35:52-61.

5. Grundy SM, Benjamin IJ, Burke GL, et al. Diabetes and cardiovascular disease: a statement for health professionals from the American Heart Association. Circulation. 1999;100:1134-46.

6. Hachamovitch R, Hayes SW, Friedman JD, et al. Stress myocardial perfusion single-photon emission computed tomography is clinically effective and cost effective in risk stratification of patients with a high likelihood of coronary artery disease (CAD) but no known CAD. J Am Coll Cardiol. 2004;43:200-8.

7. Janand-Delenne B, Savin B, Habib G, et al. Silent myocardial ischemia in patients with diabetes: who to screen. Diabetes Care. 1999;22:1396400 .

8. Campisi R, Di Carli MF. Assessment of coronary flow reserve and microcirculation: a clinical perspective. J Nucl Cardiol. 2004;11:3-11.

9. American Diabetes Association. Diagnosis and classification of diabetes mellitus. Diabetes Care. 2010;33 Suppl 1:S62-9.

10. American Diabetes Association. Standards of medical care in diabetes - 2007. Diabetes Care. 2007;30 Suppl 1:S4-41.

11. Berman DS, Abidov A, Kang X, et al. Prognostic validation of a 17-segment score derived from a 20-segment score for myocardial perfusion SPECT interpretation. J Nucl Cardiol. 2004;11:414-23.

12. Le Feuvre C. Coronary artery disease in patients with diabetes. Presse Med. 2009;38:964-72.

13. Bax JJ, Bonow RO, Tschöpe $\mathrm{D}$, et al. The potential of myocardial perfusion scintigraphy for risk stratification of asymptomatic patients with type 2 diabetes. J Am Coll Cardiol. 2006;48:754-60.

14. Zellweger MJ. Prognostic significance of silent coronary artery disease in type 2 diabetes. Herz. 2006;31:240-5.

15. Zellweger MJ, Hachamovitch R, Kang X, et al. Threshold, incidence, and predictors of prognostically high-risk silent ischemia in asymptomatic patients without prior diagnosis of coronary artery disease. J Nucl Cardiol. 2009;16:193-200. 
16. Yildirimtürk O, Kiliçgedik M, Tuğcu A, et al. The relationship of microalbuminuria with left ventricular functions and silent myocardial ischemia in asymptomatic patients with type 2 diabetes. Turk Kardiyol Dern Ars. 2009;37:91-7.

17. Agarwal AK, Singla S, Singla S, et al. Prevalence of coronary risk factors in type 2 diabetics without manifestations of overt coronary heart disease. J Assoc Physicians India. 2009;57:135-42.

18. Fateh-Moghadam S, Reuter T, Htun P, et al. Stress echocardiography for risk stratification of asymptomatic patients with type 2 diabetes mellitus. In J Cardiol. 2009;131:288-90.

19. Scholte AJ, Schuijf JD, Kharagjitsingh AV, et al Different manifestations of coronary artery disease by stress SPECT myocardial perfusion imaging, coronary calcium scoring, and multislice CT coronary angiography in asymptomatic patients with type 2 diabetes mellitus. J Nucl Cardiol. 2008;15:503-9.

20. Avignon A, Sultan A, Piot C, et al. Osteoprotegerin: a novel independent marker for silent myocardial ischemia in asymptomatic diabetic patients. Diabetes Care. 2007;30:2934-9.

21. Chico A, Tomás A, Novials A. Silent myocardial ischemia is associated with autonomic neuropathy and other cardiovascular risk factors in type 1 and type 2 diabetic subjects, especially in those with microalbuminuria. Endocrine. 2005;27 213-7.

22. Mamcarz A, Chmielewski M, Braksator W, et al. Factors influencing cardiac complications in pa- tients with type-2 diabetes mellitus and silent myocardial ischaemia: five-year follow-up. Pol Arch Med Wewn. 2004;112:1433-43.

23. Al-Attar AT, Mahussain SA, Sadanandan S. Cardiac tests in asymptomatic type 2 diabetics. Med Princ Pract. 2002;11:171-5.

24. Langer A, Freeman MR, Josse RG, et al. Detection of silent myocardial ischemia in diabetes mellitus. Am J Cardiol. 1991;67:1073-8.

25. Zellweger MJ, Hachamovitch R, Kang X, et al. Prognostic relevance of symptoms versus objective evidence of coronary artery disease in diabetic patients. Eur Heart J. 2004;25:543-50

26. Rajagopalan N, Miller TD, Hodge DO, et al. Identifying high-risk asymptomatic diabetic patients who are candidates for screening stress singlephoton emission computed tomographic imaging. J Am Coll Cardiol. 2005;45:43-9.

27. Vinik AI, Maser RE, Mitchel BD, et al. Diabetic autonomic neuropathy. Diabetes Care. 2003;26: 1553-79.

28. Valensi P, Pariès J, Attali JR, et al. Cardiac autonomic neuropathy in diabetic patients: influence of diabetes duration, obesity, and the microangiopathic complications - the French multicenter study. Metabolism. 2003;52:815-20.

29. Lee KH, Jang HJ, Kim YH, et al. Prognostic value of cardiac autonomic neuropathy independent and incremental to perfusion defects in patients with diabetes and suspected coronary artery disease. Am J Cardiol. 2003;92:1458-61.

30. Doubell AF. Managing the asymptomatic diabetic patient with silent myocardial ischaemia Cardiovasc J S Afr. 2002;13:189-93.

31. Barthelemy O, Le Feuvre C, Timsit J. Silent myocardial ischemia screening in patients with diabetes mellitus. Arq Bras Endocrinol Metabol. 2007;51:285-93.

32. Young LH, Wackers FJ, Chyun DA, et al. Cardiac outcomes after screening for asymptomatic coronary artery disease in patients with type 2 diabetes: the DIAD study: a randomized controlled trial. JAMA. 2009;301:1547-55.

33. Beller GA. Noninvasive screening for coronary atherosclerosis and silent ischemia in asymptomatic type 2 diabetic patients: is it appropriate and cost-effective? J Am Coll Cardiol. 2007;49:1918 23

34. Ueyama T, Takehana K, Maeba H, et al. Prognostic value of normal stress-only technetium- $99 \mathrm{~m}$ myocardial perfusion imaging protocol. Comparison with standard stress-rest protocol. Circ J. 2012;76:2386-91.

35. Chang SM, Nabi F, Xu J, et al. Normal stress-only versus standard stress/rest myocardial perfusion imaging: similar patient mortality with reduced radiation exposure. J Am Coll Cardiol. 2010; 55:221-30.

36. Lima RSL, Fonseca LMB. Evaluation of myocardial viability. Radiol Bras. 2010;43(5):v-vi.

37. Moraes RF, Meneghetti JC, Barroso AA. Lowdose dobutamine myocardial perfusion scintigraphy in the identification of viable myocardium. Radiol Bras. 2010;43:281-7. 